\title{
Prevalence, Determinants And Associated Risk Of Potentially Inappropriate Prescribing For Older Adults In Qatar: A National Retrospective Study
}

This article was published in the following Dove Press journal:

Clinical Interventions in Aging

\author{
Ameena Alyazeedi' \\ Ahmed Fouad Algendy $\mathbb{D}^{2}$ \\ Mohamed Sharabash (iD ${ }^{3}$ \\ Ahmed Karawia ${ }^{4}$ \\ 'Pharmacy Department, Rumailah \\ Hospital, Hamad Medical Corporation, \\ Doha, Qatar; ${ }^{2}$ Clinical Pharmacy, \\ Geriatric Department, Rumailah \\ Hospital, Hamad Medical Corporation, \\ Doha, Qatar; ${ }^{3}$ Clinical Pharmacy, \\ Rumailah Hospital, Hamad Medical \\ Corporation, Doha, Qatar; ${ }^{4}$ Pharmacy \\ Informatics and Inventory, Rumailah \\ Hospital, Hamad Medical Corporation, \\ Doha, Qatar
}

Purpose: To assess the prevalence and associated risk of potentially inappropriate prescribing (PIP) in older adults.

Methods: This was a national 3-year retrospective study of outpatient older adults exposed to potentially inappropriate medication (PIM) or polypharmacy. We used the Beers Criteria 2019 list to identify PIM to be avoided in older adults. We define moderate polypharmacy (MoP) and major polypharmacy (MaP) as using 6-10 or $>10$ chronic medications, respectively. Determinants of PIP included patients' demographics, lab results, medications, comorbidities, and home healthcare services. We used Chi-square (for categorical variables), Unpaired $t$-test and ANOVA (for continuous variables as applicable) to assess the association of these determinants with PIP. Univariate followed by multivariate logistic regression models were used to get the crude and adjusted odds ratios of exposure to PIM or polypharmacy within patients who had emergency department (ED) admissions, bone fractures, falls, or constipation, compared to those who had not.

Results: 3537 patients were included. $62.6 \%, 40.4 \%$, and $27.2 \%$ were exposed to PIM, MoP and MaP, respectively. Determinants of PIP included age, gender, ethnicity, weight, kidney function, sodium levels, hypertension, diabetes, heart failure, CAD, and home healthcare services (all with $p$-value $<0.05$ ). PIM was associated with risk of ED admission, bone fracture and constipation with adjusted OR ( $p$-values) of $1.27(0.002), 1.33(0.005)$, and 1.40 $(<0.001)$, respectively. MoP was associated with the risk of ED admission, bone fracture, and constipation, with adjusted OR ( $p$-values) of $1.27(0.012), 1.34(0.019)$, and $1.47(<0.001)$, respectively. MaP was associated with a higher risk of ED admission, bone fracture, falls, and constipation with adjusted OR ( $p$-values) of $1.46(0.001), 1.59(0.002), 1.39(0.023)$, and $2.07(<0.001)$, respectively.

Conclusion: PIP is common and is associated with an increased risk of poor clinical outcomes in older adults.

Keywords: polypharmacy, Beers 2019, falls, constipation, emergency admission, fracture

\section{Introduction}

\section{Background}

It is well established that our world is aging. The population aged over 60 years is increasing quicker than all younger age groups. By 2050, 16\% of the world will be 65 years or over, compared to $9 \%$ in $2019 .{ }^{1}$ As an individual grows older, the routine of prescribing medications increases due to the co-existence of multiple co-morbidities, with each requiring one or more medication to be managed which led to the emergence of the phenomenon called polypharmacy. ${ }^{2}$
Correspondence: Ahmed Fouad Algendy Pharmacy Department, Rumailah Hospital, Hamad Medical Corporation, PO 3050, Doha, Qatar

Tel +97470094424

Fax +97444397600

Email amohamed89@hamad.qa 
Polypharmacy can be clinically defined as using any medication without appropriate indication. While for research purposes, there is no single accepted numerical definition. However, the use of more than 5 medications is a widely accepted cutoff point. ${ }^{3,4}$ Appropriate polypharmacy is the application of the related guidelines to patients with multiple chronic issues. This exposes them to multiple medications, increasing the chance for Drug-Drug Interactions (DDI) and adverse drug reactions (ADRs). ${ }^{5}$ Recently, "inappropriate polypharmacy" expression used to describe medications that introduce a significant risk of ADRs when there are lower-risk and equally or more effective alternatives available. ${ }^{6}$ It also describes the use of medicines at higher frequencies, for longer durations, than clinically indicated, or the use of multiple medicines that have recognized drug-disease interactions. ${ }^{7}$ Similarly, Potentially Inappropriate Medication (PIM) is the medication that increases the risk of ADRs when there is a safer alternative available. ${ }^{8}$ It is used to define the quality of the prescribed medications rather than the accumulated quantities.

Though PIP can be entirely appropriate in several cases amongst the young adult population, safe and effective prescribing in the elderly is challenging. This is mainly because of the age-related pathophysiological changes as enzymatic dysfunction and liver and kidney function decline that is impacting the pharmacokinetics and pharmacodynamics of medications. ${ }^{9}$ In addition, the elderly exhibits pronounced response to certain medications especially those affecting the central nervous system, so the incidence of ADRs is expected to be higher compared to the younger population. ${ }^{10}$ Having multiple chronic comorbidities, taking several medications from a variety of physicians, and having age-related pathophysiologic changes, older adults are at increased risks of accumulating polypharmacy and PIM and hence ADRs, DDIs, and hospitalization. ${ }^{11-14,17,18}$ Generally, polypharmacy and PIM representing two arms of Potentially Inappropriate Prescribing (PIP) that may cause harm to older adults.

The appropriate prescription should maximize efficacy, safety, minimize cost, and respect patients' preferences. ${ }^{15}$ In a retrospective national study $(\mathrm{n}=338$ 801) in Ireland, PIM represents $9 \%$ of the overall pharmaceutical cost in patients $\geq 70$ years in 2007. ${ }^{16}$ As a result, many screening tools have been published to identify inappropriate prescribing in the older population in the USA and Europe in the past few decades. 46 assessment tools were available worldwide between 1991 and $2013 .{ }^{19}$ One of the most validated tools is "Beers Criteria" for PIM in older adults that were started in 1991 and was prepared later in 2012, 2015 and 2019 by the American Geriatric Society. ${ }^{20}$

Researches on PIM and polypharmacy among older adults are common in the western world. ${ }^{21}$ However, there are little researches in the Middle East, and particularly nothing published in Qatar about community-dwelling older adults. Population older than 60 years in Qatar is expected to increase from $2.3 \%$ of the total population in 2015 to $19.8 \%$ in $2050 .{ }^{22}$ In addition, this study becomes more feasible after years of using a national Electronic Health Record (EHR) for all the governmental primary and secondary healthcare centers and hospitals.

\section{Objective}

The objective of this study is to determine the prevalence of both PIM and polypharmacy amongst the elderly population followed-up in the main geriatric hospital in Qatar at the outpatient clinics and explore their significant determinants and associated risks in a period of 3 years.

\section{Methods}

\section{Study Design}

This was a national registry-based retrospective study of outpatient older adults who exposed to PIP as compared to those who did not expose for the last 3 years. The main exposure was PIP which involved PIM and polypharmacy in two independent analyses.

For the PIM evaluation, the sample was divided into two groups depending on PIM exposure. However, for polypharmacy, patients were categorized into three groups according to the maximum number of chronic systemic medications used simultaneously. Determinants of PIP included patients' age, gender, weight, Body Mass Index (BMI), kidney function estimation, ethnicity, the number of chronic systemic medications and comorbidities, hypertension, diabetes, heart failure, Coronary Artery Disease (CAD), schizophrenia/bipolar disorder, dementia, and the need for home healthcare services.

A univariate logistic regression used to identify the association of PIP use with the incidence of Emergency Department (ED) admission, bone fracture, falls, and constipation. Then, the determinants were included in a multivariate logistic regression model to get the adjusted odds ratio.

\section{Setting And Participants}

All the older adults attending geriatrics outpatient clinics in the central geriatric hospital (Rumailah Hospital, Hamad 
Medical Corporation, Qatar) through 2018 are screened for this study. Patients who did not complete the age of 65 years by Jan 1, 2018, were excluded from the extracted report. Patients demographics, medications, results, diagnoses and admissions through the past 3 years since Jan. 2016, were extracted from the EHR system (Cerner system ${ }^{\circledR}$ ). The extracted data were validated, de-identified and listed in excel sheets by three healthcare professionals including medical informatics specialist.

\section{Variables And Data}

The main exposures were PIM use and polypharmacy, which were used as categorical variables. The medication was considered as PIM if it is found in the 2nd table of the 2019 Beers list of medications that should be avoided in older adults independent of their indication or patient comorbidities (Supplementary Table S1). Our list of medications included 85 PIMs in Anticholinergic, Anti-infective, Cardiovascular, Central nervous system, Endocrine, Gastrointestinal, Pain, and genitourinary classes. Certain medications required special criteria to be considered as PIM such as Nitrofurantoin (if estimated Glomerular Filtration eGFR $<30 \mathrm{~mL} / \mathrm{min} / \mathrm{m} 2$ ), Dronedarone (if permanent atrial fibrillation or severe or recently decompensated heart failure), Digoxin (if dosages $>0.125 \mathrm{mg} /$ day), Antipsychotics (if not for schizophrenia or bipolar disorder, or for short-term use as antiemetic during chemotherapy), Androgens/Growth hormone (if no confirmed hormonal deficiency with clinical symptoms), Vaginal Estradiol (if $>25 \mu \mathrm{g}$ twice weekly) Metoclopramide (if no gastroparesis), Proton-pump inhibitors PPI (if scheduled daily $>56$ days), and NSAIDs (if $>28$ days and no gastroprotective agent). All these criteria were applied by a geriatric healthcare professional and reviewed by a geriatric clinical pharmacist through screening of clinical notes for the patients using these medications. The patient was considered in the PIM exposure group if he used at least one medication from the previous Beers list of medications between Jan 1, 2016, and Dec 31, 2018, otherwise, he was considered as unexposed to PIM.

We identified polypharmacy using chronic systemic medications only. Therefore, we excluded medications dispensed for less than 4 weeks through the study period, used topically or duplicate orders. Patients who did not use more than five chronic medications were considered "without polypharmacy" while those who used 6-10 or more than 10 chronic medications were considered as MoP or MaP, respectively.

Patients' characteristics extracted were age, nationality, gender, weight, height, serum creatinine, medications,
Diagnoses, medical problems, and admissions. CAD identified as any form of angina or myocardial infarction. Chronic kidney disease (CKD) stages were classified as per the National Kidney Foundation system using eGFR which was calculated using the 2009 Chronic Kidney Disease Epidemiology Collaboration (CKD-EPI) equation. ${ }^{23,24} \mathrm{We}$ considered dialysis patient as stage G5 regardless of the serum creatinine level. BMI was calculated using the common formula of weight in $\mathrm{kg}$ divided by height in meters squared $\left(\mathrm{kg} / \mathrm{m}^{2}\right)$ and classified into 6 categories. ${ }^{25}$ Patients from 53 countries were grouped into 6 ethnicities depending on the geographical distribution as Gulf, North African, Black African, Asian, Eastern Mediterranean, and white ethnicities (Supplementary Table S2). Total anticholinergic and CNS active medications used per patient identified through the comprehensive lists mentioned in the 2019 Beers criteria. $^{20}$

\section{Bias}

CKD staging used only the value of eGFR which is a good indicator for kidney function but it is not the only CKD staging criteria. ED admission may result from comorbidities that are causing polypharmacy, as patients who are taking fewer medications are expected to be healthier. However, testing other outcomes like falls, fractures, and constipation besides adjusting our results to the most common comorbidities and disabilities that can cause ED admissions, may show us the negative health outcomes associated with using many medications. In addition, falls documentation accuracy depends on many factors that are not monitored in this study such as the attending physician specialty, patient socioeconomic factors, and severity of fall. This may explain the poor association that we found between falls and PIM or MoP.

\section{Study Size And Power}

We used convenience sampling by including all patients aged 65 years or more and visited any of the outpatient clinics in our central geriatric hospital through 2018. Using the included sample size of 3537, this study was at least powered at $85.1 \%$ to detect an odds ratio of 1.25 . This was calculated using a Chi-square test for two-sample proportions, assuming that $30 \%$ of the control group is exposed to the risk and at an alpha level of 0.05 using a two-sided confidence interval.

\section{Statistical Methods}

PIM prevalence was the percentage of patients using at least one PIM in the total sample. The number of chronic 
medications was categorized into three categories of $0-5$, 6-10 and $>10$, so the prevalence of each polypharmacy group is calculated separately.

The difference in PIP determinants was tested using the chi-square test for categorical variables and summarized as numbers and percentages. For continuous variables in the two PIM groups $t$-test was used and summarized as mean and standard deviation (Table 1) and the difference in means was listed in the Supplementary Table S3. ANOVA was used for continuous variables in the 3 polypharmacy groups (Table 1). Bonferroni multiple-comparison test was used after ANOVA if it resulted in significant p-value to check for which groups were different (Supplementary Table S4).

Continuous variables were used in their original continuous form, while age, BMI, eGFR, and sodium were categorized into clinical subgroups as well. Numbers of PIMs, chronic medications, comorbidities, CNS-active medications, anticholinergics, and ED admissions treated as continuous variables. Missing data count and percentage are listed under each relevant variable in Table 1.

A univariate logistic regression used to identify the association of PIP with emergency admission, bone fracture, falls and constipation all as categorical outcomes compared to those without PIP. Then, the determinants found in the baseline characteristic results were included in a multivariate logistic regression model to get the adjusted odds ratio. The analysis was done initially using complete case analysis approach, however, and because of the expected missing data in lab results (weight, sodium, and creatinine), a final multivariate regression model was done after using multiple imputations (MI) to replace missing observations. Multiple imputations included all the available observations for the patient. We listed the three odds ratios along with their 95\% Confidence Intervals $(95 \% \mathrm{CI})$ and p-values in Table 2. STATA 15 was used for all the statistical work.

\section{Results}

\section{Sample Characteristics And PIP Determinants}

In total, 3539 older adults visited the outpatient clinics during 2018, identified by their unique medical record number MRN and name, of them, only two patients were excluded because of incomplete identifiers. Thus, 3537 patients have been included, 1947 (55.0\%) out of them were males. The average age was 72.8 years with 1464
(41.4\%) aged 65-70, while $218(6.2 \%)$ aged 85 years or older. The average weight was $79.7 \mathrm{~kg}$ and BMI was 29 $\mathrm{kg} / \mathrm{m}^{2}$ with 1079 (30.5\%) had obesity classes I-III. 1982 $(56.0 \%)$ were Gulf ethnicity, 520 (14.7\%) Asians, 541 (15.3\%) Eastern Mediterraneans, 227 (6.4\%) North Africans, 170 (4.8\%) Black Africans, and only 89 (2.5\%) were from White ethnicity. 859 (24.3\%) classified as G3aG5 CKD stages with eGFR $<60 \mathrm{~mL} / \mathrm{min} / 1.73 \mathrm{~m}^{2}$. Every patient had on average 2 comorbidities, 8 chronic medications, 1.4 anticholinergics, 1.3 CNS-active medications, 0.9 PIM, and 1.2 ED admissions. In addition, 2129 $(60.2 \%)$ were diabetic, 2847 (80.5\%) hypertensive, 440 (12.4\%) with heart failure, 1008 (28.5\%) with CAD, 381 $(10.8 \%)$ with dementia, $27(0.8 \%)$ with Schizophrenia or bipolar disorder, 2366 (66.9\%) had an infection, while 571 (16.1\%) needed a special home healthcare service.

\section{PIM}

$2215(62.6 \%)$ of the total sample had at least one PIM, $39.3 \%$ of them aged $65-69$ years compared to $6 \%$ aged 85 years or more, $50.7 \%$ males, $60.8 \%$ Gulf nationals, $35.1 \%$ with obesity classes I-III, and $27.7 \%$ were G3a-G5 CKD stages with eGFR $<60 \mathrm{~mL} / \mathrm{min} / 1.73 \mathrm{~m}^{2}$.

PIMs were more prevalent in females than males (68.7 vs $57.7 \%, \mathrm{p}<0.001)$ and in patients aged $80-84$ years $(69.6 \%$, $\mathrm{p}=0.003$ ) than other age groups without significant difference in age per years $(\mathrm{p}=0.082)$. PIM prevalence increased persistently over the BMI groups from $60.6 \%$ in the underweight group to $77.4 \%$ in the class III obesity group with $2.6 \mathrm{~kg}$ higher weight (95\% CI 1.21-3.98) and $1.14 \mathrm{~kg} / \mathrm{m}^{2}$ higher BMI (95\% CI 0.63-1.65) than those without PIM. In addition, it increased persistently over the CKD stages from $61.8 \%$ in the stage G1 to $76 \%$ in the stage G5 with $4.1 \mathrm{~mL} / \mathrm{min} / 1.73 \mathrm{~m}^{2}$ lower eGFR (95\% CI 2.38-5.81) and $9.05 \mu \mathrm{mol} / \mathrm{L}$ higher serum creatinine (95\% CI 3.38-14.72) than those without PIM. PIM was more common as $72.2 \%(\mathrm{p}=0.013)$ in patients with hyponatremia compared to 65.3 and $50 \%$ in patients with normonatremia and hypernatremia, respectively. PIM prevalence in different ethnicities were 68.0, 61.9, 60.8, 55, 44.1, and $36.0 \%(p<0.001)$ in the Gulf, Eastern Mediterranean, North African, Asian, Black African and White groups, respectively. Compared to undiagnosed patients, PIM was more prevalent in patients diagnosed with diabetes (70.8 vs $50.2 \%, \mathrm{p}<0.001$ ), hypertension (67.7 vs $41.9 \%, \mathrm{p}<0.001)$, heart failure (77.1 vs $60.6 \%, \mathrm{p}<0.001)$, CAD (74.3 vs $58 \%$, $\mathrm{p}<0.001$ ), infection (68.6 vs $51.0 \%, \mathrm{p}<0.001)$ and patient under home healthcare service ( 70.6 vs $61.1 \%, \mathrm{p}<0.001)$, 
Table I Characteristics Of PIM And Polypharmacy Groups And Their Prevalence Within Different Determinants

\begin{tabular}{|c|c|c|c|c|c|c|c|c|c|c|c|c|c|}
\hline \multirow[t]{3}{*}{ Determinants } & \multicolumn{5}{|l|}{ PIM } & \multicolumn{7}{|c|}{ Polypharmacy } & \multirow{3}{*}{\begin{tabular}{|l|} 
Total \\
n (\%)
\end{tabular}} \\
\hline & \multicolumn{2}{|l|}{ With } & \multicolumn{2}{|c|}{ Without } & \multirow[t]{2}{*}{ p-value } & \multicolumn{2}{|c|}{ 0-5 Meds } & \multicolumn{2}{|c|}{ 6-10 Meds } & \multicolumn{2}{|c|}{$>10$ Meds } & \multirow[t]{2}{*}{ p-value } & \\
\hline & $\mathbf{n}$ & $\%$ & $\mathbf{n}$ & $\%$ & & $\mathbf{n}$ & $\%$ & $\mathbf{n}$ & $\%$ & $\mathbf{n}$ & $\%$ & & \\
\hline Total & 2215 & 62.6 & 1322 & 37.4 & & 1144 & 32.3 & 1430 & 40.4 & 963 & 27.2 & & $3537(100)$ \\
\hline Age in years, $\mu$ and SD & 72.9 & 6.7 & 72.5 & 7.3 & 0.082 & 72.3 & 7.3 & 72.9 & 6.9 & 73.1 & 6.7 & 0.024 & $72.8(6.9)$ \\
\hline Age group, years & & & & & 0.003 & & & & & & & 0.001 & \\
\hline $65-69$ & 871 & 59.5 & 593 & 40.5 & & 527 & 36 & 571 & 39.0 & 366 & 25.0 & & | 464 (4|.4) \\
\hline $70-74$ & 564 & 64.4 & 312 & 35.6 & & 275 & 31.4 & 363 & 41.4 & 238 & 27.2 & & $876(24.8)$ \\
\hline $75-79$ & 382 & 64.0 & 215 & 36 & & 175 & 29.3 & 236 & 39.5 & 186 & 31.2 & & $597(16.9)$ \\
\hline $80-84$ & 266 & 69.6 & 116 & 30.4 & & 95 & 24.9 & 169 & 44.2 & 118 & 30.9 & & $382(10.8)$ \\
\hline$\geq 85$ & 132 & 60.6 & 86 & 39.5 & & 72 & 33 & 91 & 41.7 & 55 & 25.2 & & $218(6.2)$ \\
\hline Gender & & & & & $<0.001$ & & & & & & & $<0.001$ & \\
\hline Female & 1092 & 68.7 & 498 & 31.3 & & 443 & 27.9 & 661 & 41.6 & 486 & 30.6 & & $1590(45.0)$ \\
\hline Male & 1123 & 57.7 & 824 & 42.3 & & 701 & 36 & 769 & 39.5 & 477 & 24.5 & & $1947(55.0)$ \\
\hline Weight in $\mathrm{Kg}, \mu$ \& SD & 80.5 & 17.8 & 77.9 & 16.8 & $<0.001$ & 76.2 & 16.7 & 78.9 & 16.4 & 83.6 & 19.1 & $<0.001$ & $79.7(17.6)$ \\
\hline Missing & 342 & 45.8 & 405 & 54.2 & & 410 & 54.9 & 225 & 30.1 & 112 & 15 & & $747(21.1)$ \\
\hline Height in $\mathrm{cm}, \mu$ \& SD & 166 & 10 & 166 & 10.1 & 0.380 & 165 & 10.3 & 166 & 9.9 & 167 & 9.8 & 0.035 & $166(10.0)$ \\
\hline Missing & 91 & 42.9 & 121 & 57.1 & & 126 & 59.4 & 66 & 31.1 & 20 & 9.43 & & $212(6.0)$ \\
\hline BMI $\left(\mathrm{kg} / \mathrm{m}^{2}\right), \mu \&$ SD & 29.4 & 6.6 & 28.3 & 6 & $<0.001$ & 28 & 6 & 29 & 6 & 30 & 7.1 & $<0.001$ & $29(6.4)$ \\
\hline Missing & 370 & 46.5 & 426 & 53.5 & & 429 & 53.9 & 248 & 31.2 & 119 & 14.9 & & $796(22.5)$ \\
\hline Classes, BMI range & & & & & $<0.001$ & & & & & & & $<0.001$ & \\
\hline Underweight, $<19$ & 40 & 60.6 & 26 & 39.4 & & 29 & 43.9 & 23 & 34.9 & 14 & 21.2 & & $66(1.9)$ \\
\hline Normal weight, 19-24.9 & 431 & 64.0 & 242 & 36 & & 197 & 29.3 & 296 & 44.0 & 180 & 26.8 & & $673(19.0)$ \\
\hline Overweight, 25-29.9 & 597 & 64.7 & 326 & 35.3 & & 249 & 27 & 409 & 44.3 & 265 & 28.7 & & $923(26.1)$ \\
\hline Obese-Class I, 30-34.9 & 467 & 70.7 & 194 & 29.4 & & 164 & 24.8 & 282 & 42.7 & 215 & 32.5 & & $661(18.7)$ \\
\hline Obese-Class II, 35-39.9 & 190 & 72.2 & 73 & 27.8 & & 52 & 19.8 & 117 & 44.5 & 94 & 35.7 & & $263(7.4)$ \\
\hline Obese- Class III, $\geq 40$ & 120 & 77.4 & 35 & 22.6 & & 24 & 15.5 & 55 & 35.5 & 76 & 49.0 & & $155(4.4)$ \\
\hline ethnicity & & & & & $<0.001$ & & & & & & & $<0.001$ & \\
\hline Asian & 286 & 55.0 & 234 & 45 & & 231 & 44.4 & 186 & 35.8 & 103 & 19.8 & & $520(14.7)$ \\
\hline Black African & 75 & 44.1 & 95 & 55.9 & & 78 & 45.9 & 64 & 37.7 & 28 & 16.5 & & $170(4.8)$ \\
\hline Gulf & 1347 & 68.0 & 635 & 32 & & 522 & 26.3 & 828 & 41.8 & 632 & 31.9 & & $1982(56.0)$ \\
\hline North African & 138 & 60.8 & 89 & 39.2 & & 80 & 35.2 & 94 & 41.4 & 53 & 23.4 & & $227(6.4)$ \\
\hline Others & 2 & 25.0 & 6 & 75 & & 7 & 87.5 & 0.0 & 0.0 & 1 & 12.5 & & $8(0.2)$ \\
\hline Eastern Mediterranean & 335 & 61.9 & 206 & 38.1 & & 172 & 31.8 & 227 & 42.0 & 142 & 26.3 & & $541(15.3)$ \\
\hline White & 32 & 36.0 & 57 & 64 & & 54 & 60.7 & 31 & 34.8 & 4 & 4.5 & & $89(2.5)$ \\
\hline S. Creatinine, umol/l, $\mu$ and SD & 100.7 & 82.9 & 91.7 & 62.5 & 0.002 & 83.8 & 45.8 & 97.4 & 79.7 & 110.7 & 91.4 & $<0.001$ & $97.6(76.7)$ \\
\hline eGFR, $\mathrm{mL} / \mathrm{min} / 1.73 \mathrm{~m}^{2}$ & 70.4 & 23.8 & 74.5 & 21.9 & $<0.001$ & 77.9 & 20.1 & 72.2 & 22.9 & 65.6 & 24.8 & $<0.001$ & $71.8(23.2)$ \\
\hline CKD stage & & & & & 0.002 & & & & & & & $<0.001$ & \\
\hline $\mathrm{GI},>90$ & 472 & 61.8 & 292 & 38.2 & & 265 & 34.7 & 332 & 43.5 & 167 & 21.9 & & $764(21.6)$ \\
\hline $\mathrm{G} 2,60-90$ & 979 & 65.1 & 524 & 34.9 & & 453 & 30.1 & 645 & 42.9 & 405 & 27.0 & & $1503(42.5)$ \\
\hline $\mathrm{G} 3 \mathrm{a}, 45-59$ & 278 & 69.2 & 124 & 30.9 & & 76 & 18.9 & 175 & 43.5 & $|5|$ & 37.6 & & $402(11.4)$ \\
\hline G3b, 30-44 & 163 & 71.8 & 64 & 28.2 & & 29 & 12.8 & 98 & 43.2 & 100 & 44.1 & & $227(6.4)$ \\
\hline G4, I5-29 & 60 & 71.4 & 24 & 28.6 & & 16 & 19.1 & 21 & 25.0 & 47 & 56.0 & & $84(2.4)$ \\
\hline G5, $<15$ & 111 & 76.0 & 35 & 24 & & 23 & 15.8 & 60 & 41.1 & 63 & 43.2 & & $146(4.1)$ \\
\hline Missing renal parameters & 152 & 37 & 259 & 63 & & 282 & 68.6 & 99 & 24.1 & 30 & 7.3 & & $411(11.6)$ \\
\hline
\end{tabular}

(Continued) 
Table I (Continued).

\begin{tabular}{|c|c|c|c|c|c|c|c|c|c|c|c|c|c|}
\hline \multirow[t]{3}{*}{ Determinants } & \multicolumn{5}{|l|}{ PIM } & \multicolumn{7}{|c|}{ Polypharmacy } & \multirow{3}{*}{$\begin{array}{l}\text { Total } \\
\text { n (\%) }\end{array}$} \\
\hline & \multicolumn{2}{|l|}{ With } & \multicolumn{2}{|c|}{ Without } & \multirow[t]{2}{*}{ p-value } & \multicolumn{2}{|c|}{ 0-5 Meds } & \multicolumn{2}{|c|}{ 6-10 Meds } & \multicolumn{2}{|c|}{$>10$ Meds } & \multirow[t]{2}{*}{ p-value } & \\
\hline & $\mathbf{n}$ & $\%$ & $\mathbf{n}$ & $\%$ & & $\mathbf{n}$ & $\%$ & $\mathbf{n}$ & $\%$ & $\mathbf{n}$ & $\%$ & & \\
\hline Sodium $\mathrm{mEq} / \mathrm{L}, \mu$ and SD & 138.3 & 3.4 & 138.8 & 3.5 & $<0.001$ & 139.1 & 3.3 & 138.6 & 3.3 & 137.7 & 3.6 & $<0.001$ & $138.5(3.4)$ \\
\hline $\begin{array}{l}\text { Sodium levels } \\
\text { Hyponatremia < I35 } \\
\text { Normonatremia } 135-145 \\
\text { Hypernatremia > } 455 \\
\text { Missing sodium }\end{array}$ & $\begin{array}{l}267 \\
1770 \\
8 \\
170\end{array}$ & $\begin{array}{l}72.2 \\
65.3 \\
50 \\
38.6\end{array}$ & $\begin{array}{l}103 \\
941 \\
8 \\
270\end{array}$ & $\begin{array}{l}27.8 \\
34.7 \\
50 \\
61.4\end{array}$ & 0.013 & $\begin{array}{l}68 \\
767 \\
8 \\
301\end{array}$ & $\begin{array}{l}18.4 \\
28.3 \\
50 \\
68.4\end{array}$ & $\begin{array}{l}142 \\
1176 \\
5 \\
107\end{array}$ & $\begin{array}{l}38.4 \\
43.4 \\
31.3 \\
24.3\end{array}$ & $\begin{array}{l}160 \\
768 \\
3 \\
32\end{array}$ & $\begin{array}{l}43.2 \\
28.3 \\
18.8 \\
7.27\end{array}$ & $<0.001$ & $\begin{array}{l}370(10.5) \\
27 \mid 1(76.6) \\
16(0.5) \\
440(12.4)\end{array}$ \\
\hline $\begin{array}{l}\text { Medications, } \mu \text { and SD } \\
\text { PIMs, } \mu \text { and SD } \\
\text { CNS active meds, } \mu \text { and SD } \\
\text { Anticholinergics, } \mu \text { and SD } \\
\text { Comorbidities, } \mu \text { and SD }\end{array}$ & $\begin{array}{l}9.7 \\
1.5 \\
1.67 \\
1.8 \\
2.4\end{array}$ & $\begin{array}{l}4.2 \\
0.8 \\
2.6 \\
1.8 \\
1.2\end{array}$ & $\begin{array}{l}4.7 \\
0 \\
0.7 \\
0.9 \\
1.7\end{array}$ & $\begin{array}{l}3.7 \\
0 \\
1.8 \\
1.2 \\
1.3\end{array}$ & $\begin{array}{l}<0.001 \\
- \\
<0.001 \\
<0.001 \\
<0.001\end{array}$ & $\begin{array}{l}2.6 \\
0.4 \\
0.7 \\
0.9 \\
1.3\end{array}$ & $\begin{array}{l}1.9 \\
0.6 \\
1.7 \\
1.2 \\
1.2\end{array}$ & $\begin{array}{l}7.9 \\
1 \\
1.2 \\
1.4 \\
2.3\end{array}$ & $\begin{array}{l}1.4 \\
0.9 \\
2 \\
1.5 \\
1\end{array}$ & $\begin{array}{l}13.7 \\
1.5 \\
2.3 \\
2.1 \\
2.9\end{array}$ & $\begin{array}{l}2.8 \\
1 \\
3.2 \\
2 \\
1.1\end{array}$ & $\begin{array}{l}- \\
<0.001 \\
<0.001 \\
<0.001 \\
<0.001\end{array}$ & $\begin{array}{l}8(4.7) \\
0.9(1) \\
1.3(2.4) \\
1.4(1.7) \\
2(1.3)\end{array}$ \\
\hline Diabetes mellitus & & & & & $<0.001$ & & & & & & & $<0.001$ & \\
\hline $\begin{array}{l}\text { No } \\
\text { Yes }\end{array}$ & $\begin{array}{l}707 \\
1508\end{array}$ & $\begin{array}{l}50.2 \\
70.8\end{array}$ & $\begin{array}{l}701 \\
621\end{array}$ & $\begin{array}{l}49.8 \\
29.2\end{array}$ & & $\begin{array}{l}817 \\
327\end{array}$ & $\begin{array}{l}58 \\
15.4\end{array}$ & $\begin{array}{l}465 \\
965\end{array}$ & $\begin{array}{l}33.0 \\
45.3\end{array}$ & $\begin{array}{l}126 \\
837\end{array}$ & $\begin{array}{l}9.0 \\
39.3\end{array}$ & & $\begin{array}{l}1408(39.8) \\
2129(60.2)\end{array}$ \\
\hline Hypertension & & & & & $<0.001$ & & & & & & & $<0.001$ & \\
\hline $\begin{array}{l}\text { No } \\
\text { Yes }\end{array}$ & $\begin{array}{l}289 \\
1926\end{array}$ & $\begin{array}{l}41.9 \\
67.7\end{array}$ & $\begin{array}{l}401 \\
921\end{array}$ & $\begin{array}{l}58.1 \\
32.4\end{array}$ & & $\begin{array}{l}504 \\
640\end{array}$ & $\begin{array}{l}73 \\
22.5\end{array}$ & $\begin{array}{l}152 \\
1278\end{array}$ & $\begin{array}{l}22.0 \\
44.9\end{array}$ & $\begin{array}{l}34 \\
929\end{array}$ & $\begin{array}{l}4.9 \\
32.6\end{array}$ & & $\begin{array}{l}690(19.5) \\
2847(80.5)\end{array}$ \\
\hline Heart failure & & & & & $<0.001$ & & & & & & & $<0.001$ & \\
\hline $\begin{array}{l}\text { No } \\
\text { Yes }\end{array}$ & $\begin{array}{l}1876 \\
339\end{array}$ & $\begin{array}{l}60.6 \\
77.1\end{array}$ & $\begin{array}{l}1221 \\
101\end{array}$ & $\begin{array}{l}39.4 \\
23\end{array}$ & & $\begin{array}{l}1093 \\
51\end{array}$ & $\begin{array}{l}35.3 \\
11.6\end{array}$ & $\begin{array}{l}1291 \\
139\end{array}$ & $\begin{array}{l}41.7 \\
31.6\end{array}$ & $\begin{array}{l}713 \\
250\end{array}$ & $\begin{array}{l}23.0 \\
56.8\end{array}$ & & $\begin{array}{l}3097(87.6) \\
440(12.4)\end{array}$ \\
\hline CAD & & & & & $<0.001$ & & & & & & & $<0.001$ & \\
\hline $\begin{array}{l}\text { No } \\
\text { Yes }\end{array}$ & $\begin{array}{l}1466 \\
749\end{array}$ & $\begin{array}{l}58 \\
74.3\end{array}$ & $\begin{array}{l}1063 \\
259\end{array}$ & $\begin{array}{l}42 \\
25.7\end{array}$ & & $\begin{array}{l}1019 \\
125\end{array}$ & $\begin{array}{l}40.3 \\
12.4\end{array}$ & $\begin{array}{l}1014 \\
416\end{array}$ & $\begin{array}{l}40.1 \\
41.3\end{array}$ & $\begin{array}{l}496 \\
467\end{array}$ & $\begin{array}{l}19.6 \\
46.3\end{array}$ & & $\begin{array}{l}2529(71.5) \\
1008(28.5)\end{array}$ \\
\hline Infection & & & & & $<0.001$ & & & & & & & $<0.001$ & \\
\hline $\begin{array}{l}\text { No } \\
\text { Yes }\end{array}$ & $\begin{array}{l}592 \\
1623\end{array}$ & $\begin{array}{l}51 \\
68.6\end{array}$ & $\begin{array}{l}579 \\
743\end{array}$ & $\begin{array}{l}49.4 \\
31.4\end{array}$ & & $\begin{array}{l}549 \\
595\end{array}$ & $\begin{array}{l}47 \\
25.2\end{array}$ & $\begin{array}{l}423 \\
1007\end{array}$ & $\begin{array}{l}36.1 \\
42.6\end{array}$ & $\begin{array}{l}199 \\
764\end{array}$ & $\begin{array}{l}17 \\
32.3\end{array}$ & & $\begin{array}{l}I 17 \mid(33.1) \\
2366(66.9)\end{array}$ \\
\hline Dementia & & & & & 0.106 & & & & & & & 0.611 & \\
\hline $\begin{array}{l}\text { No } \\
\text { Yes }\end{array}$ & $\begin{array}{l}1962 \\
253\end{array}$ & $\begin{array}{l}62.2 \\
66.4\end{array}$ & $\begin{array}{l}1194 \\
128\end{array}$ & $\begin{array}{l}37.8 \\
33.6\end{array}$ & & $\begin{array}{l}1029 \\
115\end{array}$ & $\begin{array}{l}32.6 \\
30.2\end{array}$ & $\begin{array}{l}1269 \\
161\end{array}$ & $\begin{array}{l}40.2 \\
42.3\end{array}$ & $\begin{array}{l}858 \\
105\end{array}$ & $\begin{array}{l}27.2 \\
27.6\end{array}$ & & $\begin{array}{l}3156(89.2) \\
381(10.8)\end{array}$ \\
\hline Schizophrenia/bipolar & & & & & 0.217 & & & & & & & 0.942 & \\
\hline $\begin{array}{l}\text { No } \\
\text { Yes }\end{array}$ & $\begin{array}{l}2195 \\
20\end{array}$ & $\begin{array}{l}62.5 \\
74.1\end{array}$ & $\begin{array}{l}1315 \\
7\end{array}$ & $\begin{array}{l}37.5 \\
25.9\end{array}$ & & $\begin{array}{l}1136 \\
8\end{array}$ & $\begin{array}{l}32.4 \\
29.6\end{array}$ & $\begin{array}{l}1419 \\
11\end{array}$ & $\begin{array}{l}40.4 \\
40.7\end{array}$ & $\begin{array}{l}955 \\
8\end{array}$ & $\begin{array}{l}27.2 \\
29.6\end{array}$ & & $\begin{array}{l}3510(99.2) \\
27(0.8)\end{array}$ \\
\hline Homecare services & & & & & $<0.001$ & & & & & & & $<0.001$ & \\
\hline No & 1812 & 61.1 & 1154 & 38.9 & & 1021 & 34.4 & 1174 & 39.6 & 771 & 26.0 & & $2966(83.9)$ \\
\hline Yes & 403 & 70.6 & 168 & 29.4 & & 123 & 21.5 & 256 & 44.8 & 192 & 33.6 & & $57 \mid(16.1)$ \\
\hline ED visits, $\mu$ and SD & 1.4 & 4 & 0.9 & 1.9 & $<0.001$ & 0.7 & 1.6 & 1.2 & 2.8 & 1.8 & 5.3 & $<0.001$ & $1.2(3.4)$ \\
\hline
\end{tabular}

Notes: We used frequencies and percentages for categorical variables, otherwise the mean ( $\mu$ ) and the Standard Deviation (SD) were specified in the most left column for continuous and count variables. A dash was used if the test is not applicable.

Abbreviations: BMI, Body mass index; ED, Emergency Department; CAD, Coronary Artery Disease; CKD, Chronic Kidney Disease; CNS, Central Nervous System; eGFR, estimated Glomerular filtration rate; meds, medications; PIM, Potentially Inappropriate Medications; S, serum. 
Table 2 Crude And Adjusted Odds Ratios Of PIM And Polypharmacy Against Clinical Outcomes Before And After MI

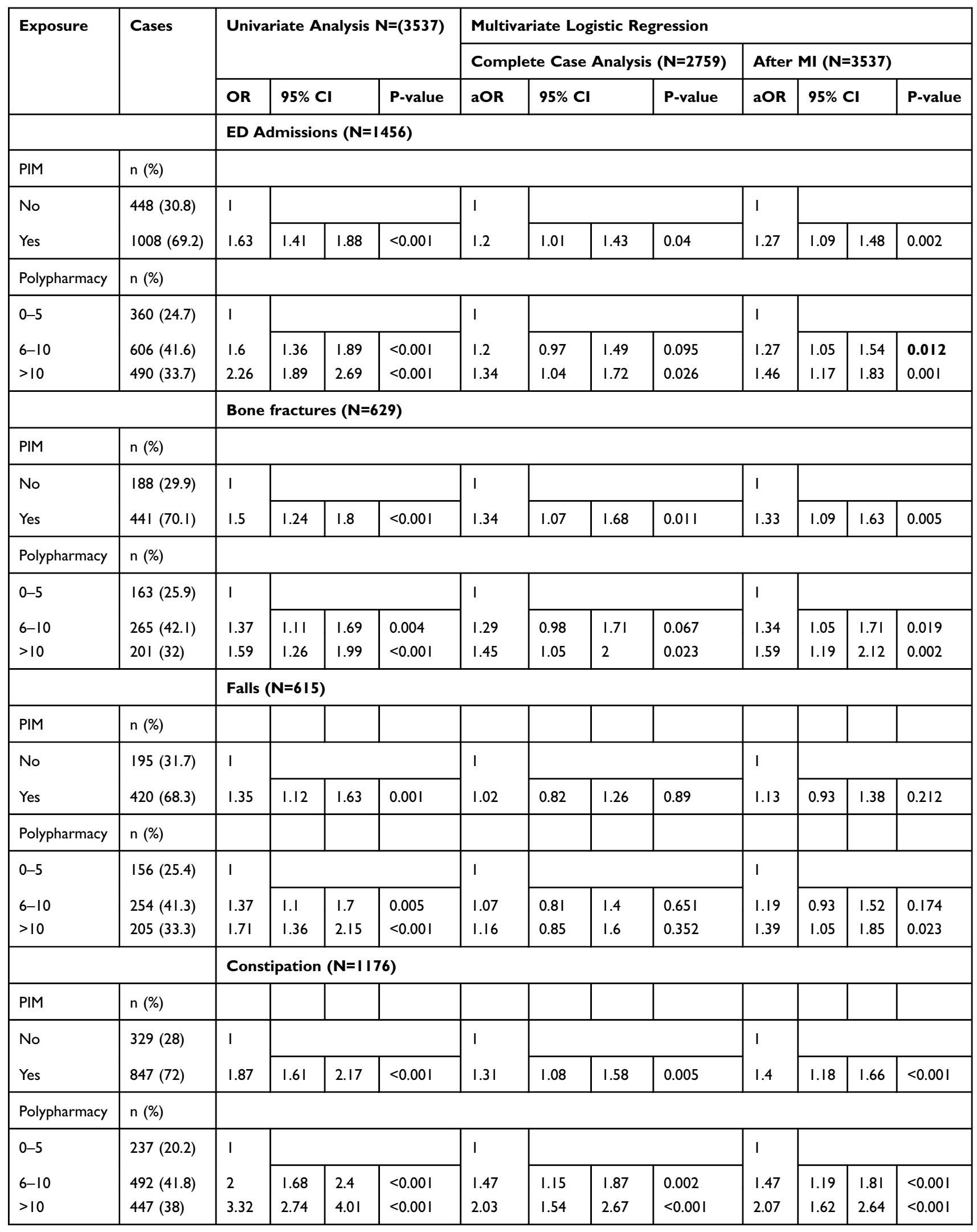

Abbreviations: $\mathrm{Cl}$, Confidence interval; Ml, Multiple Imputations; OR, Crude Odds Ratio; aOR, Odds Ratio adjusted for age, gender, ethnicity, weight, serum creatinine, sodium, infection, hypertension, diabetes, heart failure, coronary artery disease, Schizophrenia/bipolar disorder, cognitive impairment, and being under a home healthcare service. 
while no significant difference was found with dementia or schizophrenia/bipolar disorders.

PIM group also had 0.7 chronic comorbidities $(95 \%$ CI, $0.60-0.77$ ), 5 chronic medications (95\% CI, 4.7-5.3), 1 CNS-active medication (95\% CI, 0.8-1.1), 0.9 anticholinergic medication (95\% CI, 0.8-1.0), and $0.5 \mathrm{ED}$ admissions (95\% CI, 0.30-0.77) more than those without PIM (Supplementary Table S3).

\section{Polypharmacy}

Overall $2393(67.7 \%)$ of the total sample used $>5$ chronic medications, 1430 (40.4\%) used 6-10 while 963 (27.2\%) used $>10$ medications. $39.2 \%$ of the overall polypharmacy aged 65-69 years compared to $6.1 \%$ aged 85 years or more, $52.1 \%$ males, $61 \%$ Gulf nationals, $35.1 \%$ with obesity classes I-III, and $29.9 \%$ were G3a-G5 CKD stages with eGFR $<60 \mathrm{~mL} / \mathrm{min} / 1.73 \mathrm{~m} 2$.

MoP was slightly more prevalent in females than males (41.6 vs $39.5 \%, \mathrm{p}<0.001)$ and in patients aged $80-84$ years $(44.2 \%, \mathrm{p}=0.001)$ than other age groups without significant difference in age per years in the Bonferroni post-ANOVA test. It was more prevalent in obese-class II patients $(44.5 \%, \mathrm{p}<0.001), 2.7 \mathrm{~kg}$ higher weight $(p=0.002)$ and $0.9 \mathrm{~kg} / \mathrm{m}^{2}$ higher BMI $(p=0.011)$ than those without polypharmacy. Although there was a fluctuating pattern of MoP over CKD stages, MoP patients had $5.8 \mathrm{~mL} / \mathrm{min} / 1.73 \mathrm{~m}^{2}$ lower eGFR $(\mathrm{p}<0.001)$ and $13.6 \mu \mathrm{mol} / \mathrm{L}$ higher serum creatinine (95\% CI 3.3814.72) than those without polypharmacy. They had 0.5 $\mathrm{mEq} / \mathrm{L}$ lower sodium $(\mathrm{p}=0.004)$ than those without polypharmacy (Supplementary Table S4). MoP was 42.0, 41.8, 41.4, 37.7, 35.8, and 34.8\% $(\mathrm{p}<0.001)$ in the Eastern Mediterranean, Gulf, North African, Black African, Asian, and White groups, respectively. Compared to patients without polypharmacy, MoP was more prevalent in patients diagnosed with diabetes $(45.3$ vs $33.0 \%, \mathrm{p}<0.001)$, hypertension $(44.9$ vs $22 \%$, $\mathrm{p}<0.001), \operatorname{CAD}(41.3$ vs $40.1 \%, \mathrm{p}<0.001)$, infection (42.6 vs $36.1 \%, \mathrm{p}<0.001)$ and patient under home healthcare service $(44.8$ vs $39.6 \%, \mathrm{p}<0.001)$. MoP was less prevalent in patients with heart failure (31.6 vs $41.7 \%$, $\mathrm{p}<0.001$ ) while no significant difference was found with dementia and schizophrenia/bipolar disorders. MoP group also had one chronic comorbidity $(\mathrm{p}<0.001), 0.6$ PIMs $(p<0.001), 0.5$ CNS-active medications $(p<0.001)$, 0.5 anticholinergic medications $(\mathrm{p}<0.001)$, and 0.4 ED admissions $(\mathrm{p}<0.001)$ more than those without polypharmacy (Supplementary Table S4).
Major Polypharmacy (MaP) was more prevalent in females than males ( 30.6 vs $24.5 \%, \mathrm{p}<0.001)$ and in patients aged $75-79$ years $(31.2 \%, \mathrm{p}=0.001)$ than other age groups with a significant increase of 0.8 years $(p=0.034)$ than patients without polypharmacy in the Bonferroni postANOVA test. MaP prevalence increased persistently over the BMI groups from $21.2 \%$ in the underweight group to $49.0 \%$ in the class III obesity group with $7.4 \mathrm{~kg}$ higher weight $(\mathrm{p}<0.001)$ and $2.2 \mathrm{~kg} / \mathrm{m}^{2}$ higher BMI $(\mathrm{p}<0.001)$ than those without polypharmacy. MaP increased persistently over the CKD stages from 21.9 in the CKD stage G1 to $56 \%$ in the stage G4 (then falls to 43.2 in the stage G5) with $12.4 \mathrm{~mL} / \mathrm{min} / 1.73 \mathrm{~m}^{2}$ lower eGFR $(\mathrm{p}<0.001)$ and 26.9 $\mu \mathrm{mol} / \mathrm{L}$ higher serum creatinine $(\mathrm{p}<0.001)$ than those without polypharmacy. The MaP was more common as $43.2 \%$ $(\mathrm{p}<0.001)$ in patients with hyponatremia compared to 28.3 and $18.8 \%$ in patients with normonatremia and hypernatremia, respectively with $1.3 \mathrm{mEq} / \mathrm{L}(\mathrm{p}<0.001)$ lower sodium than those without polypharmacy. We found MaP in 31.9, $26.3,23.4,19.8,16.5$, and $4.5 \%(\mathrm{p}<0.001)$ of the Gulf, Eastern Mediterranean, North African, Asian, Black African and White groups, respectively. Compared to patients without polypharmacy, MaP was more prevalent in patients diagnosed with diabetes (39.3 vs $9.0 \%$, $\mathrm{p}<0.001$ ), hypertension (32.6 vs $4.9 \%, \mathrm{p}<0.001$ ), heart failure $(56.8$ vs $23 \%, \mathrm{p}<0.001)$, CAD (46.3 vs $19.6 \%$, $\mathrm{p}<0.001$ ), infection (32.3 vs $17.0 \%, \mathrm{p}<0.001)$ and patient under home healthcare service (33.6 vs $26.0 \%, \mathrm{p}<0.001$ ). No significant difference was found with dementia and schizophrenia/bipolar disorders. MaP group also had 1.7 chronic comorbidities $(\mathrm{p}<0.001), 1.2$ PIM $(\mathrm{p}<0.001), 1.6$ CNS-active medications $(\mathrm{p}<0.001), 1.2$ anticholinergic medications $(p<0.001)$, and one ED admission $(p<0.001)$ more than those without polypharmacy (Supplementary Table S4).

\section{Main Outcome Results}

Although we found PIM, MoP and MaP significantly associated with the four clinical outcomes in the crude analysis, these results slightly changed after adjusting for confounders that were expected to be associated with the PIP and clinical outcomes (Table 2).

\section{PIM}

Before and after MI, PIM use was significantly associated with ED admissions (Adjusted Odds Ratio "aOR" = 1.27, 95\% CI: $1.09-1.48, \mathrm{p}=0.002)$, bone fractures $(\mathrm{aOR}=1.33$, $95 \% \mathrm{CI}: 1.09-1.63, \mathrm{p}=0.005)$ and constipation $(\mathrm{aOR}=1.40$, 
95\% CI: $1.18-1.66, \mathrm{p}<0.001)$. On the other hand, there was non-significant increase in PIM with falls $(\mathrm{aOR}=1.13,95 \%$ CI: 0.93-1.38, $\mathrm{p}=0.212$ ).

\section{Polypharmacy}

After MI, Moderate Polypharmacy was significantly associated with $\mathrm{ED}$ admissions $(\mathrm{aOR}=1.27,95 \% \mathrm{CI}$ : $1.05-1.54$, $\mathrm{p}$-value $=0.012)$ and bone fractures $(\mathrm{aOR}=1.34,95 \% \mathrm{CI}$ : $1.05-1.71, \mathrm{p}=0.019$ ), while continued to be significantly associated with constipation $(\mathrm{aOR}=1.47,95 \% \mathrm{CI}$ : 1.19-1.81, $\mathrm{p}<0.001)$. It was rendered insignificant for falls before and after $\mathrm{MI}(\mathrm{aOR}=1.19,95 \% \mathrm{CI}: 0.93-1.52, \mathrm{p}=0.174)$.

Before and after MI, Major Polypharmacy was associated with significant increase in $\mathrm{ED}$ admissions $(\mathrm{aOR}=1.46,95 \%$ CI: $1.17-1.83, \mathrm{p}=0.001)$, bone fractures $(\mathrm{aOR}=1.59,95 \% \mathrm{CI}$ : $1.19-2.12, \mathrm{p}=0.002)$ and constipation $(\mathrm{aOR}=2.07,95 \% \mathrm{CI}$ : $1.62-2.64, \mathrm{p}<0.001)$. It was significant predictor for falls only after $\mathrm{MI}(\mathrm{aOR}=1.39,95 \% \mathrm{CI}: 1.05-1.85, \mathrm{p}=0.023)$.

\section{Sensitivity Analyses}

In total $778(22 \%)$ out of 3537 records were incomplete regarding variables included in our regression model. Missing data were in weight $(21.1 \%)$, serum creatinine (11.6\%), and sodium level (12.4\%). 406 (11.5\%) patients out of the sample had missing data in the three variables. Many patients with missing data had no observations as they show up only for medication refills, and do not go through the whole process of physical assessment. Those patients are expected to be healthier with a low incidence of poor clinical outcomes. Multiple imputations currently considered as a first-line technique with better accuracy and power compared to other missing data techniques. We used all the available data including outcome variables in the multiple imputations to create and analyze 20 multiply imputed datasets for each variable with missing data. Incomplete data were imputed using the default settings of the MICE 3.0 package of Van Buuren and Groothuis-Oudshoorn 2011. ${ }^{26}$ We ran the multivariate logistic regression initially and after multiple imputations. PIM was an insignificant predictor for falls before and after MI. However, it continued to be a significant predictor for ED admissions, fractures, and constipation. MoP was insignificant predictors for falls before and after MI as well. However, it was a significant predictor for ED admissions and bone fractures after MI and continued to be a significant predictor for constipation. On the other hand, MaP was a significant predictor of falls after MI, while continued to be a significant predictor for ED admissions, bone fractures and constipation after handling of missing data.

\section{Discussion}

Key Results

Our study found that older adults, who took at least one medication recommended to be avoided in the 2 nd table of Beers 2019 list, are common and have significantly higher odds to visit emergency, have bone fractures, or encounter episodes of constipation. Moreover, patients with MoP (6-10 medications) or MaP (more than 10 meds) were associated with significant risk for the four study outcome, except for falls in the MoP group. There is a clear ascending risk of ED admission, bone fracture and constipation form patients with MoP to those with MaP. Significant determinants for PIP included age, gender, ethnicity, weight, serum creatinine, sodium, and comorbidities including infection, hypertension, diabetes, heart failure, and CAD, besides being under home healthcare services.

To our knowledge, this is the first study in Qatar which investigates the relationship between PIP and poor clinical outcomes, and the first worldwide to study falls, bone fractures, constipation as potential outcomes to the use of qualitative and quantitative PIP (PIM and MoP/MaP) in such multiethnic older adults population.

Our results were consistent with reported associations with poor outcomes in previous literature. A recent study in the UK by Rawle and colleagues reported that "polypharmacy and excessive polypharmacy were associated with poorer cognitive and physical capability, in which stronger associations were found for excessive polypharmacy". 27 Another study in 2015 identified:

a dose-response relation between polypharmacy and allcause hospital admission (5-9 drugs: OR 1.34, 95\% CI $1.32-1.36 ; \geq 10$ drugs: OR $1.98,95 \%$ CI 1.94-2.03), and the use of potentially inappropriate medications was associated with a $27 \%$ increase in the risk of all-cause admission to hospital (OR 1.27, 95\% CI 1.25-1.28). ${ }^{28}$

On the other hand, a recent systematic review reported no association between regimen complexities measured by the Medication Regimen Complexity Index (MRCI) and emergency department visits. $^{29}$

The prevalence of inappropriate prescribing, and consequently its associated poor outcomes, internationally depends on the country conducting the research, and the tool used for detecting the PIM. In Belgium, the PIM screening with the Ghent Older People's Prescription community Pharmacy Screening $\left(\mathrm{GheOP}^{3} \mathrm{~S}\right)$ tool produced a prevalence of $78 \%$ for PIM in community-dwelling older 
adults. $^{30}$ Similarly in France, using La Roche list, 46.8\% of patients had at least one PIM. ${ }^{31}$ A Spanish Study using START/STOPP criteria detected at least one PIM in $76.4 \%$ of patients. ${ }^{32}$ A Danish study using MAI implicit criteria detected at least one PIM in $94.3 \%$ of patients, while an Austrian study using PRISCUS tool reported at least one PIM in $37.3 \%$ of patients. ${ }^{33,34}$ In Bahrain, PIM prevalence was $34.1 \%$ in older adults with hypertension or Diabetes mellitus. ${ }^{35}$ While in Qatar, one study reported a 35\% PIM prevalence in the home healthcare setting, using Beers criteria 2012. ${ }^{36}$ The later prevalence may be lower than our finding (PIM in $70.6 \%$ of patients under home healthcare services) because of using an older version of Beers criteria and/or increased PIM prescribing.

\section{Strengths}

The essential strengths of this study are using both qualitative (PIM) and quantitative (polypharmacy) measures of PIP, classifying polypharmacy into three levels depending only on chronic systemic medications count, adjusting for many important confounders, and using the most recent version of Beers criteria 2019 to identify PIM. Measuring PIP association with ED admission besides other expected ADRs give us higher trust in the results. In addition, the relatively large sample size enabled us to evaluate clinical outcomes among different categories of PIP without compromising the power of the study or the significance of the results. Patients' records are completely electronic that facilitate retrieval of accurate data and avoid the misinterpretation and misclassification of information.

\section{Limitations}

The impact of hidden confounding factors in considering the relationship between PIP and clinical outcomes in observational studies is a big challenge. Non-adherence to the recorded medications is a common limitation in all registry-based studies. The negative outcomes associated with PIP outlined here maybe because of unmeasured comorbidities, so further work is required to explore this. In addition, our definition of polypharmacy was based on a numerical count, which, although widely accepted, is not a cutoff point in daily clinical practice.

\section{Conclusion}

PIM, moderate and major polypharmacy are quite common in community-dwelling older adults in Qatar and associated with increased risk of ED admissions, bone fractures, and constipation. This necessitates revising the available safer alternatives in the formulary, customized geriatric training for non-geriatric prescribers, effective multidisciplinary team evaluation, and special prescribing restrictions whenever applicable. Achieving the therapeutic goals with the lowest number of medications and implementing de-prescribing strategies may improve older adults' health outcomes. Beers criteria 2019 list of PIMs may be linked to poor outcomes in older adults. Further researches are required to identify other clinical outcomes (including mortality risks) associated with different categories of PIMs and polypharmacy.

\section{Ethics And Approval}

This study got an exemption notice from IRB approval as it was a retrospective data analysis. Patients' data were extracted, de-identified, coded, processed, and stored using a special secured computer to maintain the highest patient confidentiality.

\section{Acknowledgments}

We honestly appreciate the supervision and support received from the Pharmacy and Geriatrics departments in Rumailah Hospital, Hamad Medical Corporation, in the state of Qatar.

\section{Funding}

The publication of this article was funded by the Qatar National Library.

\section{Disclosure}

None of the authors received any funds from any company or organization or has any conflict of interest regarding medications or topics discussed in this study.

\section{References}

1. United Nations. World population prospects: the 2019 revision. Available from: http://www.un.org/en/sections/issues-depth/ageing. Accessed May 20, 2019.

2. Masnoon N, Shakib S, Kalisch-Ellett L, Caughey GE. What is polypharmacy? A systematic review of definitions. BMC Geriatr. 2017;17 (1):695. doi:10.1186/s12877-017-0621-2

3. Van Farrell B, Shamji S, Monahan A, French Merkley V. Reducing polypharmacy in the elderly. Can Pharm J. 2013;146(5):243-244. doi:10.1177/1715163513499530

4. Payne RA, Avery AJ. Polypharmacy: one of the greatest prescribing challenges in general practice. Br J Gen Pract. 2011;61(583):83-84. doi:10.3399/bjgp11X556146

5. Boyd CM, Darer J, Boult C, Fried LP, Boult L, Wu AW. Clinical practice guidelines and quality of care for older patients with multiple comorbid diseases: implications for pay for performance. JAMA. 2005;294(6):716-724. doi:10.1001/jama.294.6.716 
6. Masoodi NA. Polypharmacy: to err is human, to correct divine. $\mathrm{Br} J$ Med Pract. 2008;1(1):6-9. Available from: http://www.bjmp.org/ files/sept2008/bjmp0908namasoodi.pdf.

7. Garfinkel D, Mangin D. Feasibility study of a systematic approach for discontinuation of multiple medications in older adults: addressing polypharmacy. Arch Intern Med. 2010;170(18):1648-1654. doi:10.1001/archinternmed.2010.355

8. Opondo D, Eslami S, Visscher S, et al. Inappropriateness of medication prescriptions to elderly patients in the primary care setting: a systematic review. PLoS One. 2012;7(8):e43617. doi:10.1371/journal.pone.0043617

9. Harugeri A, Joseph J, Parthasarathi G, Ramesh M, Guido S. Potentially inappropriate medication use in elderly patients: a study of prevalence and predictors in two teaching hospitals. J Postgrad Med. 2010;56(3):186-191. doi:10.4103/0022-3859.68642

10. Chang C-M, Liu P-Y-Y, Yang Y-HK, Yang Y-C, Wu C-F, Lu F-H. Use of the Beers criteria to predict adverse drug reactions among first-visit elderly outpatients. Pharmacotherapy. 2005;25(6):831-838. doi:10.1592/phco.2005.25.6.831

11. Anderson G, Horvath J. Chronic Conditions: Making the Case for Ongoing Care. 1st ed. Baltimore, MD: Johns Hopkins University; 2002.

12. Grimley Evans J, Franklin Williams T, Lynn Beattie B, Michael J-P, Wilcock GK. Oxford Textbook of Geriatric Medicine. 2nd ed. Oxford: Oxford University Press; 2003.

13. Gijsen R, Hoeymans N, Schellevis FG, Ruwaard D, Satariano WA, van Den Bos GA. Causes and consequences of comorbidity: a review. J Clin Epidemiol. 2001;54(7):661-674. doi:10.1016/s0895-4356(00) 00363-2

14. Field TS, Gurwitz JH, Harrold LR, et al. Risk factors for adverse drug events among older adults in the ambulatory setting. $J$ Am Geriatr Soc 2004;52(8):1349-1354. doi:10.1111/j.1532-5415.2004.52367.x

15. Barber N. What constitutes good prescribing? BMJ. 1995;310 (6984):923-925. doi:10.1136/bmj.310.6984.923

16. Cahir C, Fahey T, Teeling M, Teljeur C, Feely J, Bennett K. Potentially inappropriate prescribing and cost outcomes for older people: a national population study. Br J Clin Pharmacol. 2010;69 (5):543-552. doi:10.1111/j.1365-2125.2010.03628.x

17. Reich O, Rosemann T, Rapold R, Blozik E, Senn O. Potentially inappropriate medication use in older patients in swiss managed care plans: prevalence, determinants, and association with hospitalization. PLoS One. 2014;9(8):e105425. doi:10.1371/journal.pone.0105425

18. Hill-Taylor B, Sketris I, Hayden J, Byrne S, O’Sullivan D, Christie R. Application of the STOPP/START criteria: a systematic review of the prevalence of potentially inappropriate prescribing in older adults, and evidence of clinical, humanistic and economic impact. J Clin Pharm Ther. 2013;38(5):360-372. doi:10.1111/jcpt.12059

19. Kaufmann CP, Tremp R, Hersberger KE, Lampert ML. Inappropriate prescribing: a systematic overview of published assessment tools. Eur J Clin Pharmacol. 2014;70(1):1-11. doi:10.1007/s00228-013-1575-8

20. American Geriatrics Society, New York, New York. Updated AGS Beers Criteria ${ }^{\circledR}$ for potentially inappropriate medication use in older adults. J Am Geriatr Soc. 2019;67(4):674-694. doi:10.1111/jgs.15767

21. Rollason V, Vogt N. Reduction of polypharmacy in the elderly: a systematic review of the role of the pharmacist. Drugs Aging. 2003;20(11):817-832. doi:10.2165/00002512-200320110-00003
22. United Nations. World population ageing: highlights. Available from: https://www.un.org/en/development/desa/population/publications/ pdf/ageing/WPA2015_Highlights.pdf. Accessed March 14, 2019.

23. National Kidney Foundation. How to classify CKD. Available from: www.kidney.org/professionals/explore-your-knowledge/how-to-clas sify-ckd. Accessed May 15, 2019.

24. Levey AS, Stevens LA, Schmid CH, et al. A new equation to estimate glomerular filtration rate. Ann Intern Med. 2009;150 (9):604. doi:10.7326/0003-4819-150-9-200905050-00006

25. Nuttall FQ. Body mass index. Nutr Today. 2015;50(3):117-128. doi:10.1097/NT.0000000000000092

26. Buuren S, MICE: G-OK. Multivariate imputation by chained equations in R. J Stat Soft. 2011;45(3). doi:10.18637/jss.v045.i03

27. Rawle MJ, Cooper R, Kuh D, Richards M. Associations between polypharmacy and cognitive and physical capability: a british birth cohort study. J Am Geriatr Soc. 2018;66(5):916-923. doi:10.1111/ jgs. 15317

28. Lu W-H, Wen Y-W, Chen L-K, Hsiao F-Y. Effect of polypharmacy, potentially inappropriate medications and anticholinergic burden on clinical outcomes: a retrospective cohort study. CMAJ. 2015;187(4): E130-E137. doi:10.1503/cmaj.141219

29. Wimmer BC, Bell JS, Fastbom J, Wiese MD, Johnell K. Medication regimen complexity and polypharmacy as factors associated with allcause mortality in older people. Ann Pharmacother. 2016;50(2):8995. doi:10.1177/1060028015621071

30. Tommelein E, Mehuys E, van Tongelen I, et al. Community pharmacists' evaluation of potentially inappropriate prescribing in older community-dwelling patients with polypharmacy: observational research based on the $\mathrm{GheOP}^{3} \mathrm{~S}$ tool. J Public Health (Oxf). 2017;39(3):583-592. doi:10.1093/pubmed/fdw108

31. Montastruc F, Gardette V, Cantet C, et al. Potentially inappropriate medication use among patients with Alzheimer disease in the REAL. FR cohort: be aware of atropinic and benzodiazepine drugs. Eur $J$ Clin Pharmacol. 2013;69(8):1589-1597. doi:10.1007/s00228-0131506-8

32. Filomena Paci J, Garcia Alfaro M, Reedonodo Alonso FJ, Fernández SanMartín MI; Grupo Polimedicación Litoral. Inappropriate prescribing in polymedicated patients over 64 years old in primary care. Aten Primaria. 2015;47(1):38-47. doi:10.1016/j.aprim.2014.03.013

33. Bregnhoj L, Thirstrup S, Kristensen MB, Bjerrum L, Sonne J. Prevalence of inappropriate prescribing in primary care. Pharm World Sci. 2007;29(3):109-115. doi:10.1007/s11096-007-9108-0

34. Koper D, Kamenski G, Flamm M, Böhmdorfer B, Sönnichsen A. Frequency of medication errors in primary care patients with polypharmacy. Fam Pract. 2013;30(3):313-319. doi:10.1093/fampra/ cms070

35. KAJ AK, Ahmed Isa H, Veeramuthu S, Sequeira RP. Potentially inappropriate prescribing in older adults with hypertension or diabetes mellitus and hypertension in a primary care setting in Bahrain. Med Princ Pract. 2018;27(3):241-249. doi:10.1159/000488055

36. Alhmoud E, Khalifa S, Bahi AA. Prevalence and predictors of potentially inappropriate medications among home care elderly patients in Qatar. Int J Clin Pharm. 2015;37(5):815-821. doi:10.1007/s11096-0150125-0
Clinical Interventions in Aging

\section{Publish your work in this journal}

Clinical Interventions in Aging is an international, peer-reviewed journal focusing on evidence-based reports on the value or lack thereof of treatments intended to prevent or delay the onset of maladaptive correlates of aging in human beings. This journal is indexed on PubMed Central, MedLine, CAS, Scopus and the Elsevier
Bibliographic databases. The manuscript management system is completely online and includes a very quick and fair peer-review system, which is all easy to use. Visit http://www.dovepress.com/ testimonials.php to read real quotes from published authors. 